\title{
Multicomponent Maxwell-Stefan Diffusivities at Infinite Dilution
}

\author{
Thijs J.H. Vlugt ${ }^{1}$, Xin Liu ${ }^{1,2}$, André Bardow ${ }^{1,2}$ \\ ${ }^{1}$ Delft University of Technology, Process \& Energy Department, Leeghwaterstraat 44, \\ 2628CA Delft, The Netherlands, E-Mail: t.j.h.vlugt@tudelft.nl
}

${ }^{2}$ RWTH Aachen University, Lehrstuhl für Technische Thermodynamik, Schinkelstrasse 8, 52062 Aachen, Germany.

\section{Introduction}

Multicomponent diffusion plays a crucial role in (bio)chemical processes. The Maxwell-Stefan (MS) theory provides a sound framework for modelling mass transfer by diffusion. The key point in this approach is that the driving force for diffusion of component $i$ (i.e. the gradient in chemical potential $-\nabla \mu_{i}$ ) is balanced by a frictional force, resulting in the following transport equation:

$$
-\frac{1}{R T} \nabla \mu_{i}=\sum_{j=1, j \neq i}^{n} \frac{x_{j}\left(u_{i}-u_{j}\right)}{\bigoplus_{i j}}
$$

The frictional force between component $i$ and $j$ is proportional to the velocity difference $\left(u_{i}-u_{j}\right)$ and the MS diffusivity $\bigoplus_{i j}$ acts as an inverse friction coefficient. Unfortunately, MS diffusivities $\bigoplus_{i j}$ are composition dependent which needs to be taken into account in practical applications. There is therefore a considerable interest in models describing the concentration dependence of MS diffusivities. The logarithmic interpolation suggestion by Vignes is typically recommended, which was generalized to $n$-component mixtures as [1]

$$
\bigoplus_{i j}=\left(\bigoplus_{i j}^{x_{i} \rightarrow 1}\right)^{x_{i}}\left(\bigoplus_{i j}^{x_{j} \rightarrow 1}\right)^{x_{j}} \prod_{k=1, k \neq i, j}^{n}\left(\bigoplus_{i j}^{x_{k} \rightarrow 1}\right)^{x_{k}}
$$

The generalized Vignes equation requires the intrinsically ternary quantity $\bigoplus_{i j}^{X_{k} \rightarrow 1}$ which describes the friction between $i$ and $j$ when both are infinitely diluted in component $k$. This quantity is not directly accessible in experiments. Over the past decades, several models were proposed for estimating this quantity, all of the lacking a sound theoretical basis [2]. Based on linear response theory and the Onsager relations we derived an analytic expression for $\bigoplus_{i j}^{x_{k} \rightarrow 1}$. It turns out that the friction between $i$ and $j$ strongly depends on the medium $k$. 


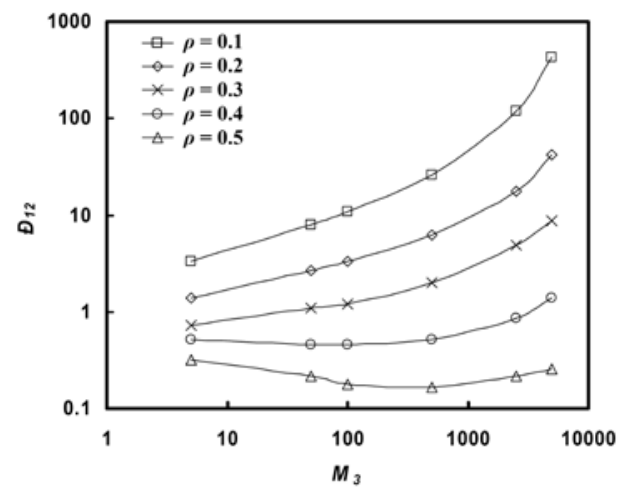

Fig. 1. Computed MS diffusivities for a ternary system in which particles interact using a WCA potential. We consider the case that components 1 and 2 are infinitely diluted in component 3 . Components only differ in molar mass. The MS diffusivity $\bigoplus_{12}$ is plotted as a function of the molar mass of component $3\left(M_{3}\right)$ for various reduced number densities $\rho$. All quantities are reported in reduced units. Molar masses of component 1 and 2 are: $M_{1}=1 ; M_{2}=1.5$.

\section{Results and Discussion}

$\bigoplus_{i j}^{X_{k} \rightarrow 1}$ can be expressed in terms of self-diffusivities and integrals over velocity cross-correlation functions [3]. As the terms latter are usually much smaller, these can often be neglected resulting in a convenient predictive model for $\bigoplus_{i j}^{x_{k} \rightarrow 1}$ in terms of binary and pure-component self-diffusivities:

$$
\bigoplus_{i j}^{X_{k} \rightarrow 1} \approx \frac{D_{\text {self }, i}^{x_{k} \rightarrow 1} D_{\text {self }, j}^{x_{k} \rightarrow 1}}{D_{\text {self }, k}^{x_{k} \rightarrow}}
$$

Molecular Dynamics (MD) simulations are used to validate the assumptions made in Eq.(2). In Fig. 1, we plot the MS diffusivity at infinite dilution for a ternary WCA system for various reduced densities. Clearly, MS diffusivities are not constants. In this system, the predictions using Eq. (2) fall within the symbol sizes and are therefore in excellent agreement with MD simulations. For associating fluids, corrections to Eq.(2) need to be taken into account as the cross-correlations can no longer be neglected.

\section{Conclusion}

We derived a model for predicting the MS diffusivity $\bigoplus_{i j}^{x_{k} \rightarrow 1}$. The inverse friction between $i$ and $j$ strongly depends on the medium (here: $k$ ). Our model provides an explanation why the MS diffusivity describing the friction between adsorbed components in a microporous material is usually very large [4]; the denominator of Eq.(2) vanishes. Eq. (2) provides a rigorous and convenient closure to the generalized Vignes equation (Eq.(1)) for the prediction of multicomponent diffusivities.

\section{References}

[1] J.A. Wesselingh, R. Krishna, Elements of mass transfer, Ellis Hoewoord: Chichester, 1990

[2] X. Liu, T.J.H. Vlugt, A. Bardow, Fluid Phase Equilibria (2011), 301, 110-117.

[3] X. Liu, A. Bardow, T.J.H. Vlugt, Ind. Eng. Chem. Res. (2011), 50, 4776-4782.

[4] R. Krishna, J.M. van Baten, Chem. Eng. Sci (2009), 64, 870-882. 